\title{
Skin Ulcers: A Sign of Disseminated Tuberculosis
}

\author{
Guven Tekbas MD, Abdurrahman Abakay MD, Abdullah Cetin Tanrikulu MD, Ugur Firat MD, \\ Faysal Ekici MD, Zeki Dostbil MD, and Bilal Sula MD
}

\section{Introduction}

Tuberculosis (TB) is still an important health problem worldwide. The estimated incidence is 4.6 cases of TB per 100,000 persons in the United States. ${ }^{1}$ According to the World Health Organization, the incidence of TB in Turkey is approximately $25-49$ per 100,000 population. $^{2}$ Generally, TB infects the lungs, but any organ system may be infected by TB. ${ }^{3}$ We report a case of disseminated TB in a 19-year-old woman, whose major complaint was skin ulcers.

\section{Case Summary}

A 19-year-old woman was referred our hospital with complaints of non-healing skin ulcers on the chest, right shoulder (Fig. 1), right humerus (Fig. 2), and foot; and night sweats, weight loss, and fatigue, for 2 months. She had lost $3 \mathrm{~kg}$ in the previous month and had difficulty walking and back pain. She did not have dyspnea. The ulcers first appeared on the left foot and right shoulder. For the foot ulcer an orthopedist had prescribed ciprofloxacin and rifampicin, without any benefit. At admission to the hospital, the foot ulcer had nearly healed (Fig. 3). She had consulted a chest physician for the fistulized swelling a month prior, and she was referred to our center but was unable to come because of financial constraints. Her medical history included occasional productive cough. She was a nonsmoker, not a drug user, and had no immunosuppres-

Drs Tekbas and Ekici are affiliated with the Department of Radiology; Drs Abakay and Tanrikulu are affiliated with the Department of Pulmonology; Dr Firat is affiliated with the Department of Pathology; Dr Dostbil is affiliated with the Department of Nuclear Medicine; and Dr Sula is affiliated with the Department of Dermatology, Dicle University Hospital, Diyarbakir, Turkey.

The authors have disclosed no conflicts of interest.

Correspondence: Guven Tekbas MD, Department of Radiology, Dicle University Hospital, 21280 Diyarbakir, Turkey. E-mail: guvenmakale@ gmail.com.

DOI: $10.4187 /$ respcare.01107

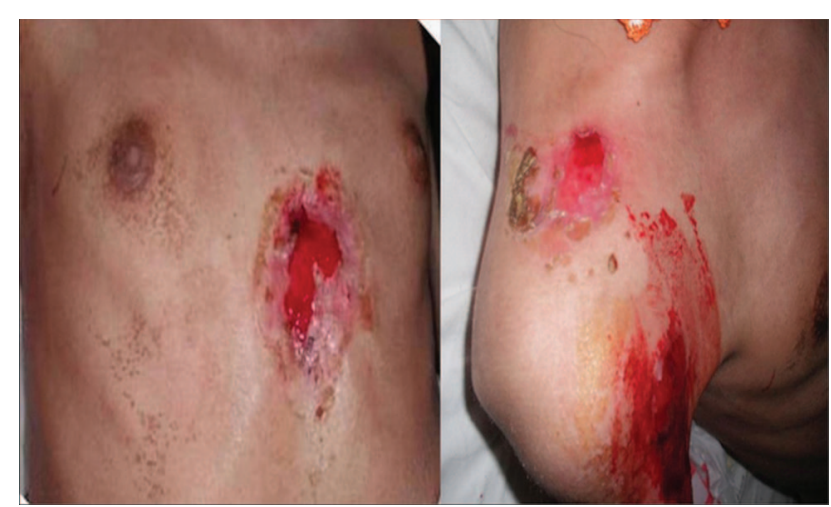

Fig. 1. Skin ulcers on the chest wall and right shoulder.

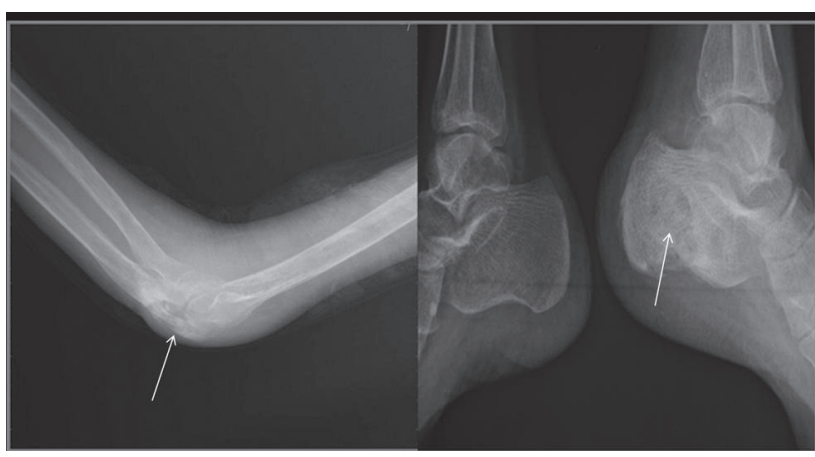

Fig. 2. Radiographs of elbow and heel show lytic areas (arrows).

sive diseases (eg, human immunodeficiency virus [HIV]). She was living with her family in poor socioeconomic conditions. She could not remember any exposure to persons with TB.

On physical examination the ulcers were fusiform and painful. The bases of the ulcers were red and had a tendency to bleed. The edges were irregular and indurated. The anterior chest-wall ulcer had some crusted edges. The intermediate zone had a reddish-blue hue. Multiple lymph nodes were palpable in the cervical region. Her breath and cardiac sounds were normal. Her blood pressure was 100/ $80 \mathrm{~mm} \mathrm{Hg}$. There were no neurological abnormalities. Her temperature was $37.8^{\circ} \mathrm{C}$. Her erythrocyte sedimentation rate was elevated $(64 \mathrm{~mm} / \mathrm{h})$, as was her C-reactive protein 


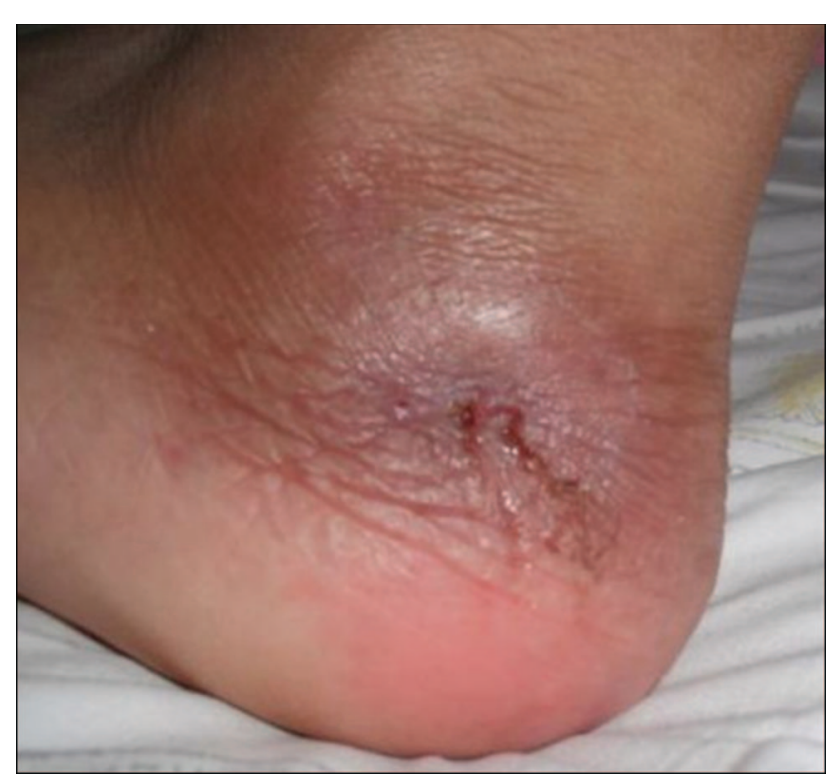

Fig. 3. Healing left foot ulcer.

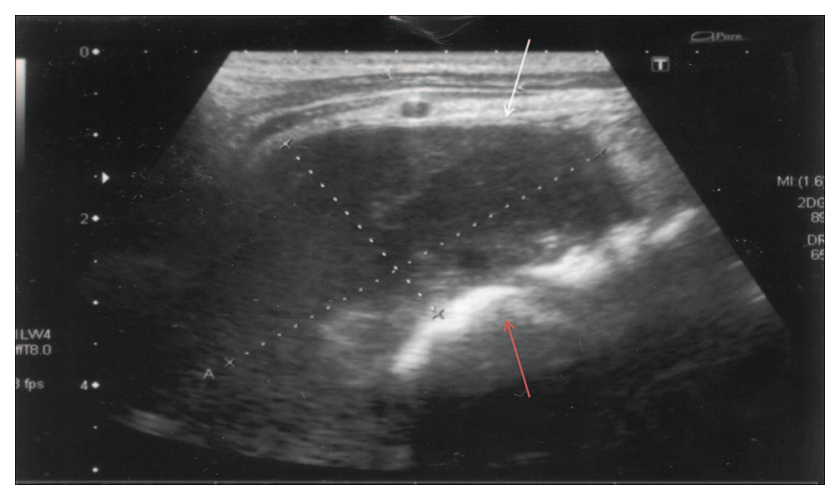

Fig. 4. Ultrasonogram shows abscess (white arrow) and vertebrae (red arrow).

$(6 \mathrm{mg} / \mathrm{dL})$. Ultrasonography of the neck revealed multiple lymph nodes in both anterior and posterior cervical chains. Additionally, hypoechoic abscesses were seen in the midcervical and deep cervical regions (Fig. 4). Computed tomogram revealed right apical lobe consolidation and a mass (density 10 Hounsfield units) anterior to the midthoracic and superior thoracic vertebrae. Magnetic resonance imaging confirmed the vertebral masses. Between the T6 and $\mathrm{C} 2$ vertebrae there was an abscess in the anterior paravertebral region, which extended up the anterior dural space and compressed the spinal cord (Fig. 5). It was surprising that she had no neurological symptoms or signs, since the abscess compressed the trachea posteriorly. Both the magnetic resonance imaging and the computed tomography showed vertebral lytic zones adjacent to the abscess. Bone scintigraphy (with ${ }^{99}$ technetium and methylene diphosphate) showed mild to moderate nonspecifically increased radioactivity at the lesion sites on the cervicotho-

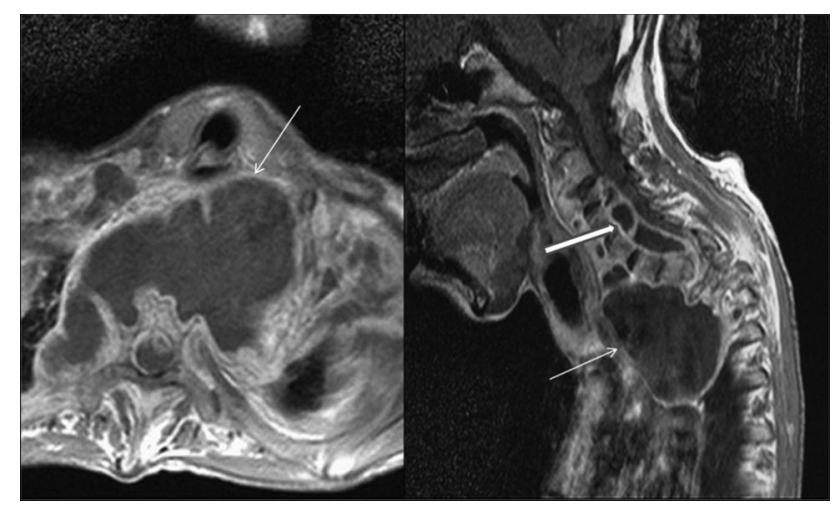

Fig. 5. Magnetic resonance imaging shows Pott abscess, lysis of the vertebral body, compression of the spine, and dural abscesses. The thick white arrow points to a dural abscess, and the thin white arrow points to a prevertebral abscess.

racic vertebrae, identified on the computed tomogram and magnetic resonance image, but no other skeletal lesions.

Excisional biopsy of the cervical lymph node revealed caseating necrosis. Acid-fast stain of the lymph node was positive. However, tissue culture was negative. These results and radiological findings supported a diagnosis of TB. We instituted anti-TB treatment (isoniazid, rifampin, ethambutol, and pyrazinamide) for the disseminated TB. She declined surgery for the abscesses. On the 20th day of therapy there was mild shrinkage of the ulcers. Her appetite improved, and weight loss and night sweats ceased. She remains on regular follow-up with her physician and is compliant with therapy.

\section{Discussion}

Disseminated TB refers to involvement of 2 or more non-contiguous sites. Dissemination can occur during primary infection or after reactivation of a latent focus/reinfection. Miliary TB is an advanced form of disseminated TB. ${ }^{4}$ However, miliary and disseminated TB are not exactly different, according to many authors, and some papers have used the terms interchangeably., ${ }^{5,6}$ Extra-pulmonary involvement in TB occurs in more than $50 \%$ of HIVinfected patients. The risk of extra-pulmonary TB and mycobacteremia increases with increasing immunosuppression, due to HIV infection, diabetes, renal or bone marrow transplantation, anti-tumor-necrosis-factor-alpha treatment, bacillus of Calmette and Guérin therapy, or idiopathic CD4+ T cell lymphopenia. ${ }^{7,8}$ Patients with HIV infection have a nearly 5-fold higher risk of extrapulmonary TB than TB patients who do not have HIV. ${ }^{1}$ Our patient had none of those risk factors.

Common extra-pulmonary TB sites include the lymph nodes, pleura, genito-urinary tract, bones, joints, meninges, and the peritoneum. ${ }^{9}$ Lymphadenitis is the most com- 
mon form of extrapulmonary TB. Most cases of lymphadenitis are localized to the cervical region; less commonly the inguinal, axillary, mesenteric, mediastinal, and intramammary regions may be involved. ${ }^{7}$ In our patient, lymphadenitis was the secondary finding and was localized to the cervical region. Cutaneous TB is rare: it constitutes about $2 \%$ of all cases of TB. ${ }^{10}$ In Turkey, among patients with TB the estimated incidence of cutaneous TB is 3.5\%. In Western countries, the 3 most common forms of cutaneous TB are scrofuloderma, lupus vulgaris, and verrucosa cutis. Scrofuloderma is the most common type of cutaneous TB in Turkey, but lupus vulgaris is the most common type worldwide. Some authors have reported that the frequencies of scrofuloderma and lupus vulgaris are equal. ${ }^{11}$

Scrofuloderma results from contiguous involvement of the skin from underlying TB in deeper structures (eg, lymph nodes or bone). Scrofuloderma and lupus vulgaris can occur after bacillus of Calmette and Guérin or purified-protein-derivative testing. The time interval between vaccination and skin-lesion development may be several months to years (mean time 1 year). Factors responsible can include the virulence of the organism, the amount of inoculum, and the inoculation technique. ${ }^{12}$ Scrofuloderma appears as firm subcutaneous nodules that ulcerate and develop sinus tracts in overlying skin. The most commonly affected areas are the neck, axillae, chest wall, and groin. The patient may have active pulmonary or pleural disease and systemic symptoms. ${ }^{13-15}$ Scrofuloderma has been most commonly associated with active pulmonary TB and lymphadenitis. ${ }^{10}$ The differential diagnosis includes actinomycosis, granuloma inguinale, lymphogranuloma venerum, and hydradenitis suppurativa. In our patient scrofuloderma was localized to the upper thoracic region, right upper extremity, and left foot. She also had consolidation in the right lung and cervical lymphadenitis. In the diagnosis of scrofuloderma, culture and purified-protein-derivative test may be useful, and a clinical history of TB is supportive. ${ }^{14}$

Lupus vulgaris usually results from re-infection of the skin, or it may develop from direct extension or hematogenous or lymphatic spread from visceral TB. Rarely, it can appear at the site of bacillus of Calmette and Guérin vaccination. Females are more commonly affected. Those located on the extremities usually occur by re-inoculation, as in verrucosa cutis. The macroscopic appearance is of slowly enlarging plaque with a slightly elevated verrucose border and central atrophy. The plaque is the result of an enlarging papule representing multiple coalescent micro-papules known as lupomes. Lupus vulgaris of the face produces scarring and facial deformity. The lesions may ulcerate at the center and cause exophytic growth, especially on the nose. Lupus vulgaris is progressive and leads to substantial function impairment and disfiguration. The most serious complication of longstanding lupus vulgaris is the devel- opment of carcinoma. Squamous-cell carcinoma is more common than basal-cell carcinoma. ${ }^{15,16}$ In the diagnosis of lupus vulgaris, histology, culture (50-60\% positive) positivity, purified-protein-derivative test, and polymerasechain-reaction test may be useful. ${ }^{14}$

Verrucosa cutis is characterized by a solitary, verrucose plaque. It is usually seen on an extremity, such as the hand or foot, and is usually painless. Verrucosa cutis is caused by mycobacterium re-inoculation in a previously exposed individual. Adults and children affected are those who have moderate immunity. Biopsy shows pseudocarcinomatous hyperplasia with poorly defined, noncaseating, tuberculous granulomata. ${ }^{14,15}$ In the diagnosis of verrucosa cutis, histology, culture, and response to treatment may be useful.

The other cutaneous manifestations of TB are chancre, gummas, acute miliary TB, and orificial TB. Primary cutaneous inoculation with $\mathrm{TB}$ in a previously uninfected host will result in a TB chancre and regional lymphadenopathy. Skin lesions develop 2-3 weeks after inoculation. Gummas are subcutaneous cold abscesses that form fistulae and ulcers. They can occur on the trunk, extremities, and head. Transmission occurs via acute hematogenous spread from a primary focus during periods of bacteremia and low resistance. Acute miliary TB occurs via hematogenous spread from a primary pulmonary focus. Most affected patients are immunocompetent. It is usually seen in children and adolescents. Purified-protein-derivative test may be negative. Orificial TB, which occurs via self-inoculation from mucosa or skin, is rare and usually affects middle-aged men. The lesions can be located anywhere in the buccal mucosa, around the anus, vulva, or penis. It is generally associated with active internal TB infection. ${ }^{14,15}$

Other common sites of disseminated TB include bones and joints. Osteoarticular TB is often the result of a hematogenous or lymphatic spread of Mycobacterium tuberculosis. ${ }^{17}$ The spine is the most common site of osseous involvement in TB and most frequently affects the upper lumbar and lower thoracic spine. ${ }^{18}$ In contrast to previously reported patients, our patient had mid-thoracic and cervical spine involvement. With TB progression there is progressive vertebral collapse with anterior wedging, leading to the characteristic angulation and gibbus formation. Extension of TB from the vertebra and disk to adjoining ligaments and soft tissues usually occurs anterolaterally. Paravertebral abscesses form early and are easily seen in the thoracic region as posterior mediastinal masses. ${ }^{19}$ In our patient the abscess had broad extension in the anterior paravertebral region of cervicothoracic vertebrae (T6 to $\mathrm{C} 2$ ), and it spread to the dural space and compressed the spinal cord. There were lytic lesions in both the left calcaneous and right olecranon, both of which are rare locations for skeletal TB. ${ }^{19,20}$ 


\section{Skin Ulcers: A Sign of Disseminated Tuberculosis}

\section{Teaching Points}

- Skin is an infrequent location for TB, so cutaneous TB could be misdiagnosed. Correct diagnosis largely depends on the clinician's experience.

- Cutaneous TB should make the clinician suspect disseminated TB. Generally, immunocompromised patients have more propensity for disseminated TB, but immunocompetent patients can also have it.

- Lupus vulgaris is generally more common than other forms of cutaneous TB in western countries, and these patients should remain on regular follow-up to watch for malignancy.

- Scrofuloderma and orificial TB are associated with active TB.

- In patients with chronic, recurrent, and non-healing ulcers, TB should be considered, especially in endemic areas.

- Cutaneous TB may be the first sign of disseminated TB.

\section{REFERENCES}

1. Kipp AM, Stout JE, Hamilton CD, Van Rie. Extrapulmonary tuberculosis, human immunodeficiency virus, and foreign birth in North Carolina, 1993-2006. BMC Public Health 2008;8:107.

2. Maartens G, Wilkinson RJ. Tuberculosis. Lancet 2007;370(9604): 2030-2043.

3. Lin JN, Lai CH, Chen YH, Lee SS, Tsai SS, Huang CK, et al. Risk factors for extra-pulmonary tuberculosis compared to pulmonary tuberculosis. Int J Tuberc Lung Dis 2009;13(5):620-625.

4. Aggarwal B, Menon B. A case with pulmonary tuberculosis, pleural effusion, miliary tuberculosis, cervical and mediastinal lymphadenopathy, tubercular arthritis, psoas abscess and severe anemia. Respir Med Extra 2007;v3-i2:79-82.

5. Mert A, Ozaras R. A terminological controversy: do disseminated and miliary tuberculosis mean the same? Respiration 2005;72(1): 113.

6. Diagnostic standards and classification of tuberculosis in adults and children. This official statement of the American Thoracic Society and the Centers for Disease Control and Prevention was adopted by the ATS Board of Directors, July 1999. This statement was endorsed by the Council of the Infectious Disease Society of America, September 1999. Am J Respir Crit Care Med 2000;161(4 Pt 1):13761395.

7. Golden MP, Vikram HR. Extrapulmonary tuberculosis: an overview. Am Fam Physician 2005;72(9):1761-1768.

8. Salliot C, Allanore Y, Lebrun A, Guerini H, Champion K, Anract P, Kahan A. Disseminated extrapulmonary tuberculosis revealed by humeral osteomyelitis with chronic unremarkable pain. Joint Bone Spine 2005;72(3):263-266.

9. Gopal R, Padmavathy BK, Vasanthi S, Jayashree K. Extra pulmonary tuberculosis - a retrospective study. Ind J Tub 2000;47:245.

10. Semaan R, Traboulsi R, Kanj S. Primary Mycobacterium tuberculosis complex cutaneous infection: report of two cases and literature review. Int J Infect Dis 2008;12(5):472-477.

11. Kivanç-Altunay I, Baysal Z, Ekmekçi TR, Köslü A. Incidence of cutaneous tuberculosis in patients with organ tuberculosis. Int J Dermatol 2003;42(3):197-200

12. Najem NM, Zadeh VB, Al-Abdulrazzaq AH, Al-Otaibi SR, Kadyan $\mathrm{S}$, Joneja M. Bacillus Calmette-Guérin vaccine-induced lupus vulgaris in a child. Acta Dermatovenerol Alp Panonica Adriat 2009; 18(4):195-197.

13. Zouhair K, Akhdari N, Nejjam F, Ouazzani T, Lakhdar H. Cutaneous tuberculosis in Morocco. Int J Infect Dis 2007;11(3):209-212.

14. Almaguer-Chávez J, Ocampo-Candiani J, Rendón A. Current panorama in the diagnosis of cutaneous tuberculosis. Actas Dermosifiliogr 2009;100(7):562-570.

15. Bravo FG, Gotuzzo E. Cutaneous tuberculosis. Clin Dermatol 2007; 25(2):173-180

16. Handog EB, Gabriel TG, Pineda RTV. Management of cutaneous. tuberculosis. Dermatol Ther 2008;21(3):154-161.

17. Monteagudo B, García-Rodríguez JF, de Las Heras C, Labandeira J, Ginarte M, Durana C, Cacharrón JM. Scrofuloderma with osteoarticular tuberculosis in the Ferrol health district. Actas Dermosifiliogr 2007;98(7):470-475

18. Harisinghani MG, McLoud TC, Shepard JA, Ko JP, Shroff MM, Mueller PR. Tuberculosis from head to toe. Radiographics 2000; 20(2):449-470.

19. Megas P, Karageorgos A, Gliatis I, Marangos M. An unusual case of olecranon tuberculosis. Orthopedics 2008;31(8):810.

20. Trikha V, Gupta V, Rastogi S, Kumar R. Tuberculosis of calcaneus: assessing treatment response by Tc-99m MDP scintigraphy. Clin Nucl Med 2004;29(8):506. 